\title{
Dimensiones de la pobreza y políticas desde una perspectiva de género
}

\author{
Irma Arriagada
}

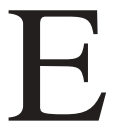

n este artículo se sostiene que la pobreza tiene carácter multidimensional y que el modo como se la define determina tanto las formas de medirla como las políticas para superarla. Tras pasar revista a diferentes definiciones, se señala que hay cierto consenso en que la pobreza es la privación de los activos y oportunidades esenciales a los que tienen derecho todos los seres humanos; se examinan conceptos relacionados con la pobreza, como los de vulnerabilidad, desigualdad, marginalidad, exclusión y discriminación, y se analizan las formas específicas que adquiere la pobreza desde una perspectiva de género. Enseguida, se examina el vínculo entre las definiciones de pobreza y las políticas que se implementan; se relacionan las políticas para enfrentar la pobreza y las políticas de género, y se elabora una tipología que distingue cuatro tipos de políticas con diversos despliegues de acciones, proyectos y programas concebidos para disminuir la pobreza de género. 


\section{Introducción}

Actualmente se reconoce que la pobreza y la desigualdad son fenómenos que aumentan y no han sido superados en la región latinoamericana: "la pobreza y la desigualdad social siguen siendo objetivos esquivos de nuestro desarrollo y han sido duramente golpeados en los últimos años por nuestra vulnerabilidad macroeconómica" (Ocampo, 2002).

Los procesos de pobreza son aspectos de fenómenos más amplios que se relacionan con los modelos y las estrategias de desarrollo puestos en marcha. Estos modelos y estrategias delimitan las opciones de apertura comercial y financiera, las políticas macroeconómicas y mesoeconómicas que son mediadas por instituciones, las normas y prácticas que en conjunto definen el acceso de los individuos y sus familias al uso y control de los recursos y, específicamente, el acceso al mercado laboral y a los ingresos. Al tradicional rezago latinoamericano en materia de pobreza y distribución de ingresos se agrega el empobrecimiento reciente de grandes sectores medios de la población latinoamericana a raíz de las crisis económicas que afectaron a la región, y con especial fuerza a algunos países, en el decenio de 1990. Además, existen evidencias acumuladas de que los efectos de estas crisis han perjudicado de diferente manera a hombres y mujeres (CEPAL, 2003 y 2004b).

Se ha llegado a cierto consenso en que la pobreza es la privación de activos y oportunidades esenciales a los que tienen derecho todos los seres humanos. La pobreza está relacionada con el acceso desigual y limitado a los recursos productivos y con la escasa participación en las instituciones sociales y políticas. Deriva de un acceso restrictivo a la propiedad, de bajos ingreso y consumo, de limitadas oportunidades sociales, políticas y laborales, de insuficientes logros educativos, en salud, en nutrición, en acceso, uso y control en materia de recursos naturales, y en otras áreas del desarrollo. Según Amartya Sen y su enfoque de las capacidades y realizaciones, una persona es pobre si carece de los recursos necesarios para llevar a cabo un cierto mínimo de actividades (Sen, 1992a y 1992b). Desai, citado en Control Ciudadano (1997), propone cinco capacidades básicas y necesarias: la capacidad de permanecer vivo y de disfrutar de una vida larga; la capacidad de asegurar la reproducción intergeneracional biológica y cultural; la capacidad de disfrutar de una vida saludable; la capacidad de interacción social (capital social) y la capacidad de tener conocimiento y libertad de expresión y pensamiento. De esta forma, la pobreza se enlaza con los derechos de las personas a una vida digna y que cubra sus necesidades básicas, es decir, con los denominados derechos económicos, sociales y culturales.

Asimismo, se sostiene que la pobreza es de naturaleza compleja, relacional y multidimensional. Las causas y características de la pobreza difieren de un país a otro y la interpretación de la naturaleza precisa de la pobreza depende de factores culturales, como los de género, raza y etnia, así como del contexto económico, social e histórico.

Este trabajo examina diversas concepciones de la pobreza y sus connotaciones desde una perspectiva de género; analiza brevemente las políticas orientadas a enfrentar la pobreza, y finalmente elabora una tipología que relaciona tales políticas con las que apuntan a la equidad de género.

\section{II}

\section{Las dimensiones múltiples de la pobreza}

Hace más de dos décadas la CEPAL definía la pobreza como "un síndrome situacional en el que se asocian el

$\square$ Una versión preliminar de este texto se presentó en la Reunión de Expertos sobre Género y Pobreza, organizada por la Unidad Mujer infraconsumo, la desnutrición, las precarias condiciones de vivienda, los bajos niveles educacionales, las

\footnotetext{
y Desarrollo de la CEPAL y por la oIt (CEPAL, Santiago, 12 y 13 de agosto del 2003).
} 
malas condiciones sanitarias, una inserción inestable en el aparato productivo, actitudes de desaliento y anomia, poca participación en los mecanismos de integración social, y quizá la adscripción a una escala particular de valores, diferenciada en alguna medida de la del resto de la sociedad" (Altimir, 1979). En esta primera definición surgen elementos que dan cuenta de las múltiples dimensiones a las que la pobreza alude: aspectos relativos a alimentación, vivienda, educación, salud, inserción en el mercado laboral y participación social, así como a otros de carácter subjetivo y simbólico y que definen también áreas diversas para la intervención de las políticas sociales.

El concepto de pobreza se ha elaborado y la pobreza se ha medido en función de carencias o necesidades básicas insatisfechas, utilizando indicadores como la ingesta de alimentos, el nivel de ingresos, el acceso a la salud, la educación y la vivienda. La CEPAL ha desarrollado una metodología para medir la pobreza sobre la base del costo de satisfacer las necesidades básicas, mediante el trazado de líneas de pobreza definidas en términos de consumo o ingreso. Este método indirecto centra las mediciones en las carencias materiales. Tiene la ventaja de que permite establecer comparaciones internacionales y efectuar una buena aproximación a la capacidad de consumo de los hogares. Según las últimas mediciones de la CEPAL para 2002, ese año vivía en la pobreza el 44\% de la población latinoamericana, porcentaje que significa 221 millones de personas, de las cuales alrededor de 97 millones eran indigentes. Para 2004 se proyecta una leve disminución en los porcentajes: la pobreza afectaría a $42,9 \%$ de la población latinoamericana y la indigencia a 18,6\% de ella, de modo que 222 millones de personas se encontrarían en situación de pobreza y 96 millones en la indigencia (CEPAL, 2003 y 2004b).

Sin embargo, el método basado en el ingreso no considera que el nivel de vida del hogar depende en parte del patrimonio acumulado ni que la distribución interna de los recursos obtenidos es desigual entre miembros de distinto sexo y edad. Además, el ingreso es una variable difícil de medir, ya que adolece de subregistros sistemáticos y presenta proporciones significativas de no respuesta. Más aún, al considerar exclusivamente ingresos corrientes en efectivo, no toma en cuenta los recursos acumulados (patrimonio) del hogar, las transferencias indirectas y subsidios del Estado en especie (servicios de salud y educación, por ejemplo). Además, con frecuencia las líneas de pobreza cortan intervalos modales de la distribución del ingreso, en los cuales se concentra mayor número de personas. En estas condiciones, las mediciones de la pobreza tienden a ser muy sensibles a cambios causados por situaciones coyunturales (incrementos de la inflación o el desempleo, por ejemplo), mostrando aumentos o disminuciones drásticas en la incidencia de la pobreza (Martínez, 2002).

En la actualidad se está tratando de incorporar en las mediciones aspectos no materiales de la pobreza, relacionados con la ampliación y fortalecimiento del capital social de la población pobre por medio de su participación en las redes sociales de intercambio: educación, trabajo, información, poder político. Este mejoramiento de los niveles de participación de la población pobre acrecienta la cultura democrática y solidaria en la sociedad, y el tiempo libre del que pueden disponer las personas para el descanso y la recreación también representa un bien valioso en situaciones en que la dificultad de generar recursos para la supervivencia lleva a alargar la jornada laboral. En suma, se han identificado seis fuentes de bienestar de las personas y hogares: i) el ingreso; ii) los derechos de acceso a servicios o bienes gubernamentales gratuitos o subsidiados; iii) la propiedad o derechos sobre activos para uso o consumo básico (patrimonio básico acumulado); iv) los niveles educativos, con las habilidades y destrezas como expresiones de la capacidad de hacer y entender; v) el tiempo disponible para la educación, el ocio y la recreación, y vi) las dimensiones que en conjunto fortalecen la autonomía de las personas. De esta forma, la pobreza queda definida en su versión más amplia por los ingresos bajos o nulos; la falta de acceso a bienes y servicios provistos por el Estado, como seguridad social y salud, entre otros; la no propiedad de una vivienda y otro tipo de patrimonio; nulos o bajos niveles educativos y de capacitación, y la carencia de tiempo libre para actividades educativas, de recreación y descanso, todo lo cual se expresa en falta de autonomía y en redes familiares y sociales inexistentes o limitadas. Sin duda que al aumentar el número de dimensiones incluidas en el concepto de pobreza se diluye la especificidad de este concepto y su medición se vuelve más compleja.

Como vemos en el gráfico 1 , cada vez más se incorporan aspectos no materiales que se relacionan con el bienestar de las personas y otros de carácter más cualitativo, como los vinculados a la vulnerabilidad, la inseguridad y la exclusión social. Por otra parte, la visión que tienen los pobres de su propia situación y la concepción de la pobreza en las distintas culturas nacionales y locales han ido adquiriendo progresivamente mayor peso como variables de análisis. La 
GRÁFICO 1

\section{Pirámide de los conceptos de pobreza ${ }^{a}$}

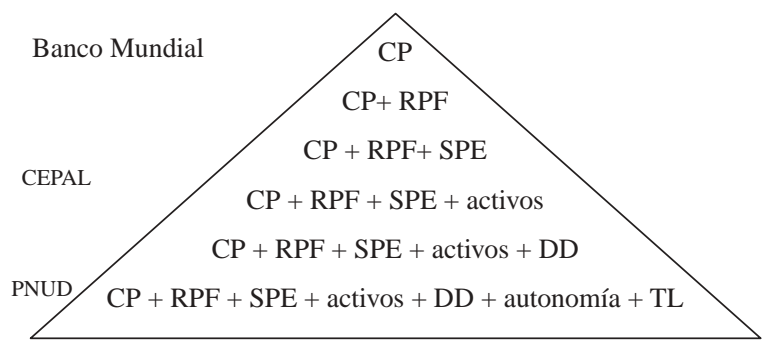

Fuente: Elaborado sobre la base de Baulch (1996) y UNIFEM (2000).

a $\mathrm{CP}=$ consumo privado $\mathrm{RPF}=$ recursos de propiedad individual y familiar; SPE = servicios y bienes provistos por el Estado; $\mathrm{DD}=$ dignidad y derechos; $\mathrm{TL}=$ tiempo libre.

premisa fundamental es que existiría un conjunto de aspectos que no son fáciles de medir en términos cuantitativos y monetarios, que influyen fuertemente en la condición de pobreza: son variables vinculadas a componentes sicosociales y culturales, y a dimensiones normativas, institucionales y cognitivas. Además, desde la filosofía se ha hecho hincapié en los aspectos éticos de la pobreza, en hacer compatibles ciertos principios de igualdad y libertad con los criterios de distribución, así como con los derechos de los pobres y con el respeto a sus preferencias (Dieterlen, 2003).

Poner a la pobreza en el centro de la preocupación de las políticas públicas puede influir fuertemente en las posibilidades de superarla, porque puede cambiar la amplitud y naturaleza de las relaciones entre los sectores pobres y aquellos que no lo son: en suma, puede modificar la amplitud de las redes sociales y el grado de asociatividad existente entre familias y grupos con capital social de unión (bonding social capi$t a l)$, capital social de puente entre grupos similares (bridging social capital) y capital social de escalera, entre grupos con distinto acceso a los recursos económicos, sociales y simbólicos (linking social capital). ${ }^{1}$ Significa hacer hincapié en el papel de las relaciones sociales de confianza, reciprocidad y cooperación, en la sustentabilidad de iniciativas comunitarias y de diversas estrategias de vida para mitigar los efectos de la pobreza. El concepto de capital social, si bien en un comienzo se utilizó para denotar la capacidad de los

${ }^{1}$ Véase un examen conceptual y metodológico del enfoque de capital social en Arriagada, Miranda y Pavez, 2004 y en Arriagada, 2003. grupos desposeídos para reaccionar frente a las crisis económicas, a las "fallas del mercado" y a los efectos de la desigualdad económica, el debate en curso ha permitido también analizar lo que ha contribuido a perpetuar la exclusión social y la reproducción de la pobreza. En el ámbito de la intervención estatal se estima que la promoción del capital social en las estrategias de desarrollo permitirá que los actores tengan mayores niveles de participación y protagonismo en la solución de sus problemas (Arriagada, I., Miranda y Pavez, 2004).

En síntesis, se podría decir que hay ciertas dimensiones básicas de la pobreza que deberán considerarse para una adecuada intervención de las políticas públicas:

- Dimensión sectorial: educación, empleo, salud, ingresos e inserción laboral, vivienda.

- Factores adscritos: el género, la raza y la etnia que cruzan las dimensiones sectoriales. También se debe considerar la edad y el ciclo de vida de las personas.

- Dimensiones territoriales. Para contribuir a la superación de la pobreza hay que trabajar a partir de las iniciativas y potencialidades existentes en los sectores pobres (capital social) y en el entorno donde ellos residen o trabajan (Raczynski, 2003).

- Dimensión familiar: es preciso tener en cuenta la etapa y el ciclo de vida familiar en que se hallan las personas, así como los intercambios económicos y la distribución del trabajo al interior de la familia. Esto podría indicar que algunos miembros de hogares no pobres (por ejemplo, mujeres sin ingresos propios) podrían ser consideradas pobres de la misma forma que hombres de hogares pobres podrían no serlo si la distribución de recursos al interior del hogar es inequitativa y ellos conservan para su propia disposición la mayor parte de sus ingresos.

\section{La pobreza desde una perspectiva de género}

La pobreza vista desde la perspectiva de género plantea que las mujeres son pobres por razones de discriminación de género. El carácter subordinado de la participación de las mujeres en la sociedad, por ejemplo, limita sus posibilidades de acceder a la propiedad y al control de los recursos económicos, sociales y políticos. Su recurso económico fundamental es el trabajo remunerado, al cual acceden en condiciones de mucha desigualdad, dada la actual división del trabajo 
por género en que las mujeres asumen el trabajo doméstico y el cuidado de los hijos de manera casi exclusiva, y la persistencia de formas tradicionales y nuevas de discriminación para el ingreso y permanencia de las mujeres en el mercado laboral. Si bien la situación en América Latina no es similar para el conjunto de mujeres, en ningún país se logra el mismo ingreso por igual trabajo entre hombres y mujeres: la existencia de una gran segmentación ocupacional, tanto vertical como horizontal, hace que las mujeres no ocupen los mismos puestos de trabajo ni accedan a los niveles superiores de las ocupaciones a la par con los hombres. A ello se yuxtaponen visiones esencialistas que atribuyen a las mujeres características que las colocan en situación de inferioridad ante los hombres, ligando su potencial reproductivo con la atribución de las tareas reproductivas.

Kabeer (1998a) señala que la pobreza puede ser vista de doble manera: como privación de la posibilidad de satisfacer necesidades básicas y como privación de los medios para satisfacerlas. Las mujeres son pobres en la medida en que no cuentan con tiempo disponible para buscar las formas más apropiadas de satisfacer sus necesidades, y una proporción importante de ellas carece de ingresos propios.

Así, en el caso de las mujeres, además de medir la pobreza en términos de ingresos adquiere relevancia medir la pobreza en términos de tiempo. Para conocer la dinámica de la pobreza es preciso analizar el concepto de tiempo, sobre todo porque parte importante del trabajo de las mujeres —el trabajo domésticono es valorizado monetariamente, pero sí puede medirse en términos de tiempo. Diversos estudios (en especial las encuestas de uso de tiempo) han mostrado que la jornada femenina es más larga que la masculina si en ella se incluye el trabajo doméstico no remunerado que realizan todas las mujeres en sus hogares. ${ }^{2}$ Asimismo, la creciente incorporación de las mujeres al mercado de trabajo no ha significado una incorporación paralela de los hombres a las actividades domésticas y de cuidado: de los hijos, de los ancianos, de otros familiares y de los enfermos.

Por lo demás, las formas tradicionales de medición de la pobreza, que privilegian el ingreso familiar,

\footnotetext{
${ }^{2}$ Las últimas encuestas de uso de tiempo realizadas en México y Uruguay en 2002 y 2003, respectivamente, indican que las mujeres mexicanas aportaban $85 \%$ del tiempo total de trabajo doméstico y los hombres $15 \%$, en tanto que los varones uruguayos responsables del hogar dedicaban un promedio de 31 horas semanales, y las mujeres responsables del hogar uno de 50 horas, al trabajo doméstico y cuidado de la familia (INEGI, 2004; Aguirre, 2004).
}

oscurecen la pobreza de los miembros menos favorecidos del hogar: mujeres, jóvenes, niños/as y ancianos/ as. Es conveniente entonces examinar el diferente uso del tiempo que hacen hombres, mujeres, jóvenes, niños/as y adultos/as mayores. Para realizar esta medición se requiere un análisis dinámico de la pobreza y de las formas en que esta aumenta o disminuye a lo largo del ciclo de vida familiar.

En lo que se refiere al trabajo en el mercado laboral, existen cuatro formas de exclusión que afectan de manera más severa a las mujeres: i) el desempleo; ii) las formas precarias de inserción laboral; iii) las formas de trabajo no remuneradas y iv) la exclusión de las oportunidades para desarrollar sus potencialidades. A estas formas de exclusión se agregan las desigualdades en las ocupaciones a las que acceden (segmentación ocupacional horizontal y vertical) y la discriminación salarial en el mercado del trabajo.

En síntesis, para analizar la pobreza desde una perspectiva de género hay que hacer visibles diversas relaciones de poder, como las ligadas a las exclusiones, desigualdades y discriminaciones de género en el mercado laboral, el reparto desigual del trabajo no remunerado, el ejercicio de la violencia física y simbólica en contra de la mujer y el diferente uso del tiempo de hombres y mujeres.

\section{Aspectos relacionales de la pobreza}

En América Latina la relación entre pobreza y desigualdad es de larga data. La evolución de ambos fenómenos en las últimas décadas ha sido desigual: aunque se ha logrado disminuir la proporción de población pobre e indigente, han persistido los niveles de desigualdad en el ingreso regional. La concentración del ingreso es una variable que incide directamente en los plazos en que sea posible superar la pobreza (PNUD, 1997). "La desigualdad (entendida como el grado de concentración y polarización de la distribución del ingreso urbano según grupos de la población), aun cuando constituye una problemática más amplia que la pobreza, constituye en el caso de América Latina un referente complementario obligado, puesto que tiene determinantes comunes y marca, además, tanto los niveles de crecimiento económico y gasto social requeridos para la erradicación de la pobreza urbana como los plazos en que puede aventurarse el logro de dicho objetivo en los distintos países" (Arriagada, C., 2000). Se estima que en América Latina, entre 1990 y 2002, ha aumentado la desigualdad en la distribución de los ingresos — medida por el coeficiente de Gini-, debido 
principalmente a la elevada proporción de ingresos que concentra el decil de hogares de ingresos más altos (CEPAL, 2004b).

Asimismo, es preciso destacar la interrelación del concepto de pobreza con los de distribución, exclusión, vulnerabilidad, discriminación y marginalidad, por citar algunos. Cuando el concepto de pobreza se define por sus dimensiones más amplias, los conceptos de exclusión y desigualdad tienden a ser incluidos en él, aun cuando es posible diferenciarlos analíticamente. Sin embargo, la distinción es importante puesto que el enfoque escogido definirá políticas y programas diferentes para enfrentar el fenómeno (cuadro 1).

En esta línea y desde un enfoque de género cabe citar las siete desigualdades específicas por género mencionadas por Amartya Sen: i) desigualdad en la mortalidad, referida a que en ciertas partes del mundo (el norte de África, Asia incluida China y el sudeste asiático) hay un índice desproporcionadamente alto de mortalidad femenina; ii) desigualdad en la natalidad,

CUADRO 1

Conceptos y dimensiones relacionados con la pobreza

\begin{tabular}{ll}
\hline Conceptos & \multicolumn{1}{c}{ Dimensiones } \\
\hline Marginalidad & $\begin{array}{l}\text { Contexto geográfico y ecológico } \\
\text { Marginalidad económica, laboral y educativa } \\
\text { Contingente de reserva de mano de obra }\end{array}$ \\
Vulnerabilidad & $\begin{array}{l}\text { Inseguridad y riesgos frente a las crisis } \\
\text { Incapacidad de respuesta }\end{array}$ \\
& $\begin{array}{l}\text { Inhabilidad de adaptación } \\
\text { Grupos objetivo y sus activos }\end{array}$ \\
Desigualdad & $\begin{array}{l}\text { Dotación de recursos desiguales } \\
\text { Justicia y equidad }\end{array}$ \\
Reglas y normas de distribución de los \\
recursos \\
Exclusión & $\begin{array}{l}\text { Quiebre de vínculos sociales, comunitarios e } \\
\text { individuales con el sistema } \\
\text { Énfasis en los procesos y su causalidad } \\
\text { múltiple }\end{array}$ \\
Interrelación de aspectos de la pobreza \\
Dimensión relacional \\
de etnia y raza \\
Institucionalidad
\end{tabular}

Fuente: Elaboración de la autora. cuando los padres prefieren hijos varones y se efectúan abortos selectivos de fetos de sexo femenino; iii) desigualdad de oportunidades básicas (prohibición o inequidad de acceso a la educación y salud básicas, al desarrollo de talentos personales o a funciones sociales en la comunidad, entre otras); iv) desigualdad de oportunidades especiales (dificultades o prohibiciones de acceso a la educación superior); v) desigualdad profesional en el acceso al mercado de trabajo y a puestos de nivel superior; vi) desigualdad en el acceso a la propiedad de bienes y tierras, y vii) desigualdad en el hogar, reflejada en la división del trabajo por género, donde las mujeres tienen a su cargo el trabajo doméstico de manera exclusiva (Sen, 2002). Asimismo, en el análisis de la pobreza no se puede ignorar el patrón medio de bienestar de la sociedad porque es este estándar el que establece las condiciones de integración, sin las cuales no hay ciudadanía.

El concepto de marginalidad surgió en el decenio de 1960 en América Latina para denotar a los grupos poblacionales que migran del campo y rodean las principales metrópolis latinoamericanas con un cinturón de pobreza. Según Nun y Marín (1968), la marginalidad se define como un proceso estructural de formación de proletariado, de nuevos pobres, y de constitución de clases sociales. La población marginal pasó a ser caracterizada como carente de infraestructura, de oportunidades educacionales y de empleo, constituyendo un ejército de reserva de mano de obra, funcional para la economía porque su presión por puestos de trabajo tendería a hacer bajar los salarios de los obreros.

La noción de vulnerabilidad se relaciona con dos dimensiones: una externa y objetiva, que se refiere a los riesgos externos a los que puede estar expuesta una persona, familia o grupo (mayor inestabilidad de los ingresos familiares, aumento de la precariedad en el mercado de trabajo reflejado en porcentajes crecientes de personas empleadas con contratos no permanentes, a tiempo parcial, sin contratos y sin seguridad social); y otra dimensión interna y subjetiva, que se refiere a la falta de recursos para enfrentar esos riesgos sin sufrir ciertas pérdidas. Este enfoque integra tres dimensiones centrales: los activos (físicos, financieros, de capital humano y social) que poseen individuos y comunidades; las estrategias de uso de esos activos, y el conjunto de oportunidades que ofrecen los mercados, el Estado y la sociedad (Moser, 1996).

El concepto de exclusión social, si bien surgió del debate europeo, tiene amplia aplicación en la región latinoamericana y específicamente frente a las nuevas situaciones de pobreza y exclusión provocadas por las 
crisis. La exclusión social se refiere a dos dimensiones: la falta de lazos sociales que vinculen al individuo con la familia, la comunidad y más globalmente con la sociedad, y la carencia de derechos básicos de ciudadanía. Lo que diferencia el concepto de exclusión social del de pobreza es que el primero se refiere a las relaciones entre aspectos de la pobreza. Los elementos de proceso que están incorporados en el debate sobre la exclusión son interesantes de considerar en la medida en que se relacionan los diversos mecanismos y tipos de exclusiones: de carácter institucional, social, cultural y territorial. Los lazos que unen al individuo con la sociedad pueden ser catalogados en tres niveles: los de tipo funcional, que permiten la integración del individuo al funcionamiento del sistema (mercado de trabajo, instituciones de seguridad social, legalidad vigente, etc.); los de tipo social, que incorporan al individuo en grupos y redes sociales (familia, grupos primarios, sindicatos, etc.), y los de tipo cultural, que posibilitan que los individuos se integren a las pautas de conducta y entendimiento de la sociedad (participación en las normas y creencias socialmente aceptadas). También puede existir exclusión espacial, vinculada al territorio y la ubicación geográfica.

La discriminación por motivos de género y etnia parte con la atribución a las personas de ciertas características de personalidad y comportamiento en razón de su sexo o del color de su piel o de otros rasgos físicos. Se basa en el esencialismo, al relacionar el sexo y los rasgos físicos externos de las personas con características socialmente construidas que segregan a estos grupos.

En términos analíticos y para los efectos de elaborar una adecuada política antipobreza es necesario distinguir entre:

- Factores de diferenciación de la pobreza, como etnia, género y generaciones, nivel educativo y ocupacional alcanzado y zona de residencia, entre otros.

- Factores de reproducción de las causas de la pobreza (transmisión intergeneracional) que se relacionan con el ciclo de vida de la persona y el ciclo de vida de la familia y con el acceso a la propiedad, al patrimonio y a los recursos económicos sociales y simbólicos.

- Consecuencias de la pobreza en lo que se refiere a pérdida de oportunidades y de bienestar y a reforzamiento de la desigualdad.

El carácter multidimensional de la pobreza obliga a que al enfocarla se tome en cuenta la diversidad de las causas que generan privación, mientras que la heterogeneidad de la pobreza destaca la importancia de reconocer las diferentes manifestaciones de ella. En esta perspectiva, para formular las políticas sociales destinadas a combatirla es esencial identificar las principales fuentes de la pobreza y la heterogeneidad de sus manifestaciones en distintos grupos o países.

\section{La pobreza como proceso y no como un esta- do de situación}

Un elemento que suele olvidarse en los análisis de la pobreza y especialmente en las políticas sociales diseñadas para erradicarla, es que la pobreza es un estado de situación que en ciertos casos se mantiene en el tiempo (pobreza estructural, pobreza dura) pero que en muchos otros varía. En los análisis tiende a vérsela como una situación estática en el tiempo. Sin embargo, la situación de pobreza puede alterarse en poco tiempo, especialmente en relación con el desempleo/ empleo, así como con los impactos de crisis económicas que pueden traducirse, entre otras cosas, en devaluación de la moneda nacional. La precariedad permanente de la situación de algunas personas, especialmente de aquéllas con menor educación y calificación, junto con nuevas formas laborales que significan inestabilidad y alta rotación en los puestos de trabajo, aumenta la vulnerabilidad ante quiebres de ingresos por desempleo. Asimismo, hay otros procesos de quiebres de ingreso que se enlazan con problemas de salud, de vejez, de disminución del ingreso debido a jubilación y retiro del mercado de trabajo, y de separación y divorcio, especialmente en el caso de mujeres cónyuges que carecen de ingresos propios.

Vemos así que comprender la dinámica de la pobreza tiene importancia crucial para establecer quienes entre los pobres pueden salir de ella, y quienes están más propensos a caer en ella debido a problemas de salud, desempleo, divorcio y/o ausencia de pareja, entre otros factores.

Las contribuciones realizadas desde el análisis de género para comprender mejor la pobreza apuntan a lo siguiente: i) ponen de relieve la heterogeneidad de la pobreza y, por lo tanto, ayudan a comprenderla mejor y a ajustar más las políticas para erradicarla; ii) permiten una nueva mirada que relaciona el comportamiento de hombres y mujeres; iii) mejoran el análisis del hogar, destacando en especial las asimetrías de poder, tanto de género como generacionales, en su interior; iv) aportan una perspectiva multidimensional de la pobreza, con el análisis de los múltiples roles desempeñados 
por hombres y mujeres; v) permiten apreciar otras discriminaciones que se combinan con las de género, como las vinculadas a edad y etnia; vi) agregan una visión dinámica del fenómeno de la pobreza al mostrar sus cambios en el tiempo, y vii) distinguen entre diversas estrategias para salir de la pobreza por género.

\section{III}

\section{Las políticas ante la pobreza}

La evolución y reformas del Estado en América Latina durante el decenio de 1990 desembocaron en una reducción de su tamaño y de sus funciones. Esto se debió al incremento de los procesos de privatización de empresas públicas; cambios en las funciones estatales, al perder el Estado el papel de productor de bienes y servicios; creciente descentralización de los servicios y también de los recursos; aparición de nuevos actores en las políticas públicas no estatales, donde los organismos no gubernamentales adquieren presencia en la entrega de servicios públicos y promueven la participación y el control comunitarios. En ese marco, también cambiaron los enfoques orientados a superar la pobreza, lo que se tradujo en más recursos, nuevas instituciones y programas, modalidades de gestión de las políticas y programas más descentralizados y participativos y con mayor injerencia de la sociedad civil, y la aplicación de nuevos instrumentos de asignación de recursos (Raczynski, 2003). En síntesis, hacia la década del 2000, junto con un aumento en los niveles de pobreza de la población, aparecen nuevas formas de gestión pública que reorientan los esfuerzos estatales hacia programas encaminados a disminuir la pobreza, en especial la extrema pobreza.

El concepto de pobreza no es nuevo en la historia. Ya a mediados del siglo XVI, en Inglaterra se propusieron leyes para combatirla (the English poor laws, dictadas en 1563, 1572, 1576, 1597 y 1601, en el período isabelino) por diversas razones: la primera, por las circunstancias económicas y por la presión de la población; luego, por cambios en la concepción (basada en el puritanismo y el protestantismo) de lo que los gobiernos podían y debían hacer a favor de los pobres y, por último, por la ambición política de controlar a quienes las elites consideraban súbditos "inferiores" (Dieterlen, 2003). Lo que es relativamente nuevo es la idea de que el Estado (así como los organismos internacionales y los organismos no gubernamentales) deben intervenir de manera continuada y sistemática para mejorar la situación de quienes viven en la pobreza y en la extrema pobreza. Más nueva aún es la comprobación de que las causas de la pobreza y la situación de pobreza misma son diferentes para mujeres y hombres y que, por lo tanto, se necesitan políticas y programas que consideren las diferencias de género en los procesos que originan y mantienen las situaciones de pobreza.

Los modelos explicativos y la forma de medir la pobreza también definen políticas para enfrentarla. Si se mide la pobreza en función de situaciones de carencia y por el método del ingreso, la consecuencia más frecuente es que se actúe sobre las carencias mediante transferencias de subsidios monetarios, cursos de capacitación laboral, pavimentación de calles, saneamiento, provisión de servicios en las áreas de educación y salud, otorgamiento de créditos bajo condiciones especiales, y otras medidas compensatorias de corto plazo (Raczynski, 2003). En cambio, las propuestas que se realizaron en la década de 1960 para disminuir la marginalidad consideraban la provisión de infraestructura urbana, la organización de los grupos marginales y la incorporación al mercado laboral como los mecanismos centrales que ayudarían a esta población a integrarse social y económicamente. Las políticas diseñadas en esa época para reducir la desigualdad social incluían redistribución de recursos por la vía impositiva y políticas sociales y económicas redistributivas, como la asignación diferenciada de recursos a la educación básica en salud y educación y las políticas de discriminación positiva a favor de individuos y grupos desfavorecidos (Raczynski, 2003).

En lo que se refiere a la discriminación por género y por etnia, las políticas propuestas en ambos casos apuntan a políticas afirmativas que favorezcan a las mujeres y a las etnias en situación más desmedrada y a políticas de deconstrucción cultural que tiendan a disminuir los prejuicios valorativos respecto de esos grupos (cuadro 2). 
CUADRO 2

Conceptos y propuestas de política relacionados con la pobreza

\begin{tabular}{|c|c|}
\hline Conceptos & Propuestas de política \\
\hline Marginalidad & $\begin{array}{l}\text { - Ampliación de servicios urbanos } \\
\text { - Organización de la población marginal } \\
\text { - Incorporación al mercado laboral }\end{array}$ \\
\hline Vulnerabilidad & $\begin{array}{l}\text { - Apoyo focalizado para fortalecer los ingresos } \\
\text { - Promoción de nuevas fuentes de ingresos o } \\
\text { subsidios } \\
\text { - Fortalecimiento de redes de protección } \\
\text { individuales, familiares o sociales }\end{array}$ \\
\hline Desigualdad & $\begin{array}{l}\text { - Redistribución de recursos } \\
\text { - Políticas sociales y económicas redistributivas } \\
\text { - Políticas de discriminación positiva }\end{array}$ \\
\hline Exclusión & $\begin{array}{l}\text { - Creación de condiciones para una ciudadanía } \\
\text { ampliada y para el pleno ejercicio de derechos } \\
\text { - Políticas sociales integrales } \\
\text { - Políticas de participación e integración } \\
\text { - Acceso a servicios institucionales }\end{array}$ \\
\hline $\begin{array}{l}\text { Discriminación } \\
\text { de género y } \\
\text { de etnia }\end{array}$ & $\begin{array}{l}\text { - Acción afirmativa } \\
\text { - Deconstrucción cultural } \\
\text { Políticas de conciliación entre trabajo } \\
\text { remunerado y familia }\end{array}$ \\
\hline
\end{tabular}

Fuente: Elaboración de la autora.

Antes de elaborar políticas sobre pobreza es importante hacer una precisión. Conviene distinguir entre, por un lado, las políticas de reducción de la pobreza, que aluden a las causas del fenómeno y a su corrección, y que se relacionan con la gestión macroeconómica, las regulaciones y las políticas institucionales para generar un desarrollo equitativo y evitar las crisis y la desigualdad y, por otro, las políticas de alivio y enfrentamiento (protección social) de la pobreza, que apuntan a disminuir el riesgo social en situaciones de crisis, es decir, buscan atenuar las consecuencias en las diversas manifestaciones de la pobreza. Entre las políticas para reducir la pobreza se pueden distinguir las orientadas a establecer derechos económicos y sociales; entre las de alivio y enfrentamiento de la pobreza se pueden incluir las de carácter compensatorio (de asistencia social) y las orientadas a proporcionar bienes y servicios sectoriales a grupos de población focalizados.

\section{Diversos enfoques de la pobreza de género}

La agenda de políticas públicas de los gobiernos y de los organismos internacionales muestra una gran diversidad de enfoques ante la pobreza y el género, los que han sido aplicados por separado o combinadamente a lo largo de las tres últimas décadas y se sintetizan a continuación: ${ }^{3}$

i) Enfoque asistencial: Incluye programas específicos para las mujeres pobres, como "grupo vulnerable". Plantea un apoyo a las mujeres teniendo en cuenta sólo sus funciones reproductivas, como esposas y amas de casa. Se aplicó de manera más generalizada en los decenios de 1970 y 1980, pero existen remanentes de él en algunos programas actuales del Banco Interamericano de Desarrollo (BID), el Banco Mundial, la Organización de las Naciones Unidas para la Agricultura y la Alimentación (FAO) y el Fondo de Población de las Naciones Unidas (UNFPA).

ii) Enfoque basado en la eficiencia: Sostiene que para lograr un mayor desarrollo sería más eficiente considerar al conjunto de la población, hombres y mujeres, en tanto personas disponibles para el mercado de trabajo, o, en una versión más elitista, por el aporte económico que la incorporación de las mujeres educadas, en su calidad de recurso humano altamente calificado, puede hacer a la economía. Lo aplican el Banco Mundial, el BID, la CEPAL, la Organización Internacional del Trabajo (огт).

iii) Enfoque basado en la equidad: Plantea que mejorar la situación de las mujeres pobres contribuiría a la equidad, puesto que hay una incidencia mayor de pobreza en los hogares encabezados por mujeres, que las mujeres reciben salarios más bajos y que su inserción laboral es precaria y segmentada, lo cual se traduce en una mala calidad de vida y dificulta el logro de equidad. Está presente en los estudios de la CEPAL y la OIT.

iv) Enfoque basado en el empoderamiento, el ejercicio de la ciudadanía social y la toma de decisiones: Según este enfoque, relativamente reciente, para que los programas dirigidos a las mujeres y otros grupos (etnias, clases) sean mejores y más eficientes hay que comprometer en su elaboración y ejecución a los propios afectados, reforzando sus capacidades mediante la organización, el mejoramiento de la autoestima, el acceso a recursos materiales y el fortalecimiento de sus redes sociales, para que puedan ejercer plenamente su ciudadanía y tomar decisiones en los planos individual, familiar y social. Lo utilizan el Programa de las Naciones Unidas para el Desarrollo (PNUD), la CEPAL, el Instituto de las Naciones Unidas para el Desarrollo Social (UNRISD).

\footnotetext{
${ }^{3}$ Véase al respecto Moser (1991).
} 
Este último enfoque hace hincapié en la promoción de políticas para superar la pobreza que tomen en cuenta todos los factores que causan la pobreza, desde los más personales — falta de autoestima y autonomía, así como la violencia que se ejerce sobre las mujereshasta los de carácter social y económico, como el acceso al empleo, la salud, la educación y los servicios técnico-financieros. Para ello propone comprometer en la elaboración y ejecución de estas políticas a los propios afectados y a actores estatales, sindicales y empresariales, y asigna un importante papel a los nuevos criterios de empoderamiento y capital social.

Entre las políticas orientadas a eliminar las desigualdades basadas en el género se encuentran, a su vez, dos conjuntos importantes: las políticas de reconocimiento de las diferencias y las políticas de redistribución, entre las cuales se hallan las de igualdad de oportunidades (Fraser, 1998 y 2000). Las políticas de reconocimiento, que Fraser denomina modelo de la identidad, se refieren a las políticas de reconocimiento de identidades injustamente desvalorizadas. Las de redistribución, en cambio, buscan una transformación o reforma socioeconómica para la solución de la injusticias de género y de etnia.

Las políticas de igualdad de oportunidades se han generalizado en el plano internacional. La igualdad de oportunidades en la tradición política liberal clásica implica que todos los individuos han de tener la misma oportunidad y que las desigualdades que se producen se deben a los distintos méritos que tienen las personas. Es decir, todos han podido utilizar las mismas oportunidades, pero como son diferentes, algunos son más capaces que otros, por lo que terminan siendo desiguales. Existen, por lo tanto, desigualdades injustas que deben ser corregidas, cuando no se ha tenido en el punto de partida las mismas oportunidades, y desigualdades que sólo expresan diferencias de mérito y que son legítimas. Con el propósito de corregir las desigualdades ilegítimas se han diseñado acciones afirmativas destinadas a establecer la igualdad de oportunidades en el punto de partida (Astelarra, 2003).

Sin embargo, como la igualdad de oportunidades de inicio no produce igualdad de resultados, desde una perspectiva democrática se hace hincapié en crear las condiciones sociales para una verdadera igualdad de oportunidades. Pero aunque se partiera de una situación inicial igualitaria, la retribución desigual tendería a perpetuarse de una generación a otra mediante las "herencias" y "sesgos de género", afectando así la igualdad de condiciones y oportunidades de las mujeres.
Las políticas de reconocimiento hacen hincapié en temas relativos a la identidad que define a los grupos discriminados. Su esfuerzo se orienta a que la cultura dominante reconozca los derechos propios de esos grupos. Una de las principales críticas a este enfoque es que podría tender a estigmatizar a tales grupos, al centrarse en la mantención de una identidad que los mantiene segregados de los demás.

Según Fraser, las políticas de reconocimiento y las de redistribución no se excluyen mutuamente.

\section{La interrelación de las políticas referidas a la pobreza y al género}

Las políticas sobre la pobreza con una perspectiva de género cruzan dos grupos importantes de políticas establecidas y puestas en marcha desde hace varias décadas en América Latina: las orientadas a eliminar las desigualdades de género y las dirigidas a disminuir la pobreza.

Al cruzar estos dos grupos se obtiene un conjunto posible de políticas destinadas a reducir la pobreza de género, con orientaciones muy dispares. El despliegue de acciones, proyectos y programas diseñados para disminuir la pobreza de género origina cuatro tipos de políticas combinadas (cuadro 3).

En el conjunto posible de políticas que se han aplicado hasta el momento podemos distinguir cuatro tipos, cuyos rasgos más estilizados se indican a continuación:

i) Políticas universales ciegas al género. Son las políticas que no distinguen entre hombres y mujeres. Se las aplicó en diversos sectores sociales, como la educación y el empleo, con el resultado de perpetuar las desigualdades de género.

ii) Políticas redistributivas y compensatorias "económicas". Este tipo de políticas se puso en marcha con sesgo más redistributivo en la década de 1960, y más compensatorio y asistencialista frente a la pobreza en el decenio de 1980. Se ha reflejado en muchos programas para las mujeres jefas de hogar que con diversos grados de éxito se han ejecutado en la región. Hacia la década de 1990, los programas dedicados a la pobreza extrema incorporaron una focalización territorial más precisa y una ejecución más descentralizada, con transferencias monetarias dirigidas principalmente a las madres, pero no han modificado la visión de las mujeres en general y de las madres en particular como personas al servicio de otros, ni se han relacionado nítidamente con las políticas sectoriales. 


\begin{tabular}{|c|c|c|c|}
\hline & \multicolumn{3}{|c|}{ Políticas contra la pobreza } \\
\hline \multirow{3}{*}{$\begin{array}{l}\text { Políticas pro equidad de } \\
\text { género }\end{array}$} & & Derechos & $\begin{array}{l}\text { Compensatorias y sectoriales/ } \\
\text { Servicios }\end{array}$ \\
\hline & De reconocimiento & $\begin{array}{l}\text { Derechos y reconocimiento } \\
\text { cultural }\end{array}$ & $\begin{array}{l}\text { De reconocimiento y } \\
\text { compensatorias }\end{array}$ \\
\hline & $\begin{array}{l}\text { De redistribución e igualdad } \\
\text { de oportunidades }\end{array}$ & $\begin{array}{l}\text { Derechos y redistribución } \\
\text { ("casillero vacío") }\end{array}$ & $\begin{array}{l}\text { De redistribución y } \\
\text { compensatorias } \\
\text { "económicas" }\end{array}$ \\
\hline
\end{tabular}

Fuente: Elaboración propia.

iii) Políticas de derechos y reconocimiento cultural. Son políticas más recientes — se han aplicado a partir del decenio de 1990 - y tienen como objetivo que se reconozcan los derechos de los grupos excluidos, entre ellos las mujeres. Incluyen principalmente medidas legislativas orientadas a la equidad de género.

iv) Políticas de redistribución y de derechos económicos sociales y culturales. A este conjunto se le denomina "casillero vacío", puesto que se trata de políticas para el futuro que no han sido aplicadas en país alguno. Si bien su diseño requiere un equilibrio cuidadoso, no son incompatibles entre ellas, y necesitan voluntad y consenso políticos más vigorosos para su puesta en marcha y ejecución. En especial, estas políticas debieran brindar más autonomía y poder a las mujeres, modificando el desequilibrio de género existente y flexibilizando los roles de género con miras a acrecentar las opciones de hombres y mujeres.

En cuanto al diseño de las políticas sociales, la heterogeneidad de la pobreza obliga a elaborar políticas que sean universales y al mismo tiempo selectivas y orientadas a grupos específicos. No es el mismo tipo de pobreza el de una mujer pobre que es madre adolescente y el de una mujer viuda sin ingresos propios, así como serán diferentes sus necesidades, sus potencialidades y los programas y políticas que deberán formularse para ellas.

Si se considera la pobreza como un proceso que va más allá de una fotografía instantánea (Kabeer, 1998b), queda en claro la importancia de formular políticas heterogéneas, flexibles y adecuadas para encarar las situaciones cada vez más diversas y cambiantes por las que atraviesa la población pobre y para el tránsito continuo de la población entre etapas de bienestar, pobreza e indigencia.

Las políticas orientadas a reducir la pobreza desde una perspectiva de género, por lo tanto, consideran los recursos individuales, familiares y sociales de hombres y mujeres; toman en cuenta el uso del tiempo de ambos géneros; apoyan el fortalecimiento de las mujeres pobres que se hallan en posiciones más débiles; tienen en cuenta la subjetividad y las diferentes necesidades de hombres y mujeres; toman en consideración la dinámica de los procesos de pobreza y por consiguiente las entradas y salidas de la pobreza, y atienden a las relaciones entre diversos aspectos de esos procesos.

\section{Evaluación de las políticas sobre pobreza des- de una perspectiva de género}

Una primera medida para evaluar las políticas de género es la de determinar sus fundamentos (éticos, culturales, económicos y otros) y comprobar si las formas de conceptualización, medición y aplicación no han desvirtuado sus objetivos centrales. Es válido preguntarse entonces si con la puesta en marcha de las políticas se avanza hacia la equidad de género o si se han ido gestando nuevas formas de desigualdad, y si en el proceso se afianza la autonomía o la dependencia respecto de los programas (Arriagada, I., 1998). Otro aspecto fundamental es el fomento de la participación activa de hombres y mujeres pobres en el diseño, ejecución y evaluación de los programas e intervenciones sociales que les afectan. Incorporar a la población en la toma de decisiones puede hacer más lento el proceso, pero a la larga redunda en un mayor compromiso con los programas, haciéndolos más sustentables, y en una notable mejora de la autoestima de la población.

Cabe recalcar la necesidad de efectuar una evaluación precisa de los cambios introducidos, para aprender de la experiencia pasada y reciente en el diseño de programas y proyectos sociales. Esto contribuirá a generar una política de género coherente con 
los objetivos iniciales planteados y a evitar que estos se desvirtúen en su ejecución. Por lo mismo, es preciso evaluar también, y de manera permanente, el impacto diferente en hombres y mujeres de las políticas sociales y económicas. Tal evaluación debe referirse a la totalidad de las políticas, ya que no ha sido ajeno a la experiencia latinoamericana que los efectos adversos de una política (por ejemplo, de una determinada política económica) deban paliarse con otras, como es el caso de los programas sociales compensatorios. Para evaluar los resultados esperados e inesperados de los programas es preciso contar con información actualizada tanto previa como posterior a las intervenciones sociales.

En especial, es necesario velar por la incorporación en la agenda pública de las políticas sobre pobreza con perspectiva de género; fortalecer la voluntad política de llevar a cabo tales políticas; ampliar la cobertura y calidad de los servicios y programas para las mujeres pobres desde una visión de género y evaluar permanentemente el grado de participación y de creciente autonomía de las personas, en especial de las mujeres que son sujeto de las políticas.

\section{Bibliografía}

Altimir, O. (1979): Dimensión de la pobreza en América Latina, serie Cuadernos de la CEPAL, No 27, Santiago de Chile.

Aguirre, R. (2004): Trabajo no remunerado y uso del tiempo. Fundamentos conceptuales y avances empíricos. La encuesta Montevideo 2003, Santiago de Chile, Comisión Económica para América Latina y el Caribe (CEPAL), por publicarse.

Arriagada, C. (2000): Pobreza en América Latina: nuevos escenarios y desafíos de políticas para el hábitat urbano, serie Medio ambiente y desarrollo, No 27, LC/L.1429-P, Santiago de Chile. Publicación de las Naciones Unidas, $\mathrm{N}^{\mathrm{o}}$ de venta: S.00.II.G.107.

Arriagada, I. (2003): Capital social: potencialidades y limitaciones de un concepto, Revista mexicana de estudios sociológicos, vol. 21, No 63, México, D.F., El Colegio de México.

(2002): Cambios y desigualdad en las familias latinoamericanas, Revista de la CEPAL, N ${ }^{\circ} 77$, LC/G.2180-P, Santiago de Chile, agosto.

(2001): Familias latinoamericanas. Diagnóstico y políticas públicas en los inicios del nuevo siglo, serie Políticas sociales, No 57, LC/L.1652-P, Santiago de Chile, Comisión Económica para América Latina y el Caribe (CEPAL), diciembre. Publicación de las Naciones Unidas, $\mathrm{N}^{\circ}$ de venta: S.01.II.G.189.

(1998): Políticas públicas y de género: una relación difícil, Revista paraguaya de sociología, año $35, \mathrm{~N}^{\circ} 101$, Asunción, Centro Paraguayo de Estudios Sociológicos, enero-abril.

Arriagada, I., F. Miranda y T. Pavez (2004): Lineamientos de acción para el diseño de programas de superación de la pobreza desde el enfoque del capital social. Guía conceptual y metodológica, serie Manuales, No 36, LC/L.2179-P, Santiago de Chile, Comisión Económica para América Latina y el Caribe (CEPAL). Publicación de las Naciones Unidas, $\mathrm{N}^{\mathrm{o}}$ de venta: S.04.II.G.106.

Arriagada, I. y C. Torres (comps.) (1998): Género y pobreza. Nuevas dimensiones, Ediciones de las Mujeres, № 26, Santiago de Chile, ISIS Internacional.

Astelarra, J. (2003): Veinte años de políticas de igualdad de oportunidades en España, presentación realizada en la Comisión Económica para América Latina y el Caribe (CEPAL).

Baulch, B. (1996): The new poverty agenda: a disputed consensus, IDS Bulletin, vol. 27, $\mathrm{N}^{\circ}$ 1, Sussex, Instituto de Estudios para el Desarrollo.

CEPAL (Comisión Económica para América Latina y el Caribe) (2003): Panorama social de América Latina, 2002-2003, LC/G.2209-P, Santiago de Chile. Publicación de las Naciones Unidas, $\mathrm{N}^{\mathrm{o}}$ de venta: S.03.II.G.185. (2004a): Caminos hacia la equidad de género en América Latina y el Caribe, LC/L.2114(CRM.9-3), documento presentado a la $9^{\text {a }}$ Conferencia Regional sobre la Mujer de América Latina y el Caribe (México, D.F., 10 al 12 de junio de 2004). (2004b): Panorama social de América Latina, 2004, LC/L.2220.P, Santiago de Chile.

Control Ciudadano (1997): Las dimensiones de la pobreza, Montevideo, Instituto del Tercer Mundo.

Desai, Meghnad (1995): Poverty and capability: towards an empirically implementable measure, en M. Desai, ed., Poverty, Famine and Economic Development, Aldershot, Edward Elgar Publishing Company.

Dieterlen, P. (2003): La pobreza: un estudio filosófico, México, D.F., Universidad Nacional Autónoma de México (UNAM)/Fondo de Cultura Económica (FCE).

Fraser, N. (1998): La justicia social en la época de la política de la identidad: redistribución, reconocimiento y participación, Contextos, $\mathrm{N}^{\circ}$ 4, año 2, Lima, Programa de Estudios de Género, Pontificia Universidad Católica del Perú, octubre.

(2000): ¿De la redistribución al reconocimiento?, New Left Review, $\mathrm{N}^{\mathrm{o}} 0$, edición en español, Londres.

Grinspun, A. (comp.) (2001): Choices for the Poor. Lessons from National Poverty Strategies, Nueva York, Programa de las Naciones Unidas para el Desarrollo (PNUD).

INEGI (Instituto Nacional de Estadística, Geografía e Informática) (2004): Encuesta Nacional sobre Uso de Tiempo, 2002, Comunicado de prensa, Aguascalientes, 8 de marzo.

Kabeer, N. (1998a): Tácticas y compromisos: nexos entre género y pobreza, en I. Arriagada y C. Torres (comps.), Género y pobre$z a$. Nuevas dimensiones, Ediciones de las Mujeres, $\mathrm{N}^{\circ} 26$, Santiago de Chile, ISIS Internacional.

(1998b): Realidades trastocadas. Las jerarquías de género en el pensamiento del desarrollo, Estudios de género, México, D.F., Paidós/Universidad Nacional Autónoma de México.

Martínez, J. (2002): Exposición en el Panel inaugural "El combate a la pobreza en las visiones estratégicas para la consolidación de un proyecto país" del Seminario CEPAL/MIDEPLAN "Perspectivas innovativas en política social" (Santiago de Chile, 29 y 30 de octubre).

Moser, C. (1991): La planificación de género en el Tercer Mundo: enfrentando las necesidades prácticas y estratégicas del género, en V. Guzmán, P. Portocarrero y V. Vargas (comps.), Una nueva lectura: género en el desarrollo, Lima, Ediciones Entre Mujeres.

(1996): Situaciones críticas: reacciones de los hogares de cuatro comunidades urbanas pobres ante la vulnerabilidad y la pobreza, serie Estudios y monografías sobre desarrollo 
ecológicamente sostenible, $\mathrm{N}^{\circ} 75$, Washington, D.C., Banco Mundial.

Nun, M. y J.C. Marín (1968): La marginalidad en América Latina, documento de trabajo, $\mathrm{N}^{\circ} 53$, Buenos Aires, Instituto Torcuato di Tella, versión preliminar.

Raczynski, D. (2003): Equidad, inversión social y pobreza. Innovar en cómo se concibe, diseña y gestiona las políticas y los programas sociales, documento presentado en el Seminario CEPAL/MIDEPLAN "Políticas innovativas de desarrollo social" (Santiago de Chile, 23 y 24 de mayo).

Ocampo, J.A. (2002): Discurso pronunciado en el Día de las Naciones Unidas, Santiago de Chile, 24 de octubre.

PNUD (Programa de las Naciones Unidas para el Desarrollo) (1997): Informe sobre desarrollo humano, 1997, Nueva York, Oxford University Press.
Sen, A. (1992a): Conceptos de pobreza, en L. Beccaria y otros, América Latina: el reto de la pobreza. Conceptos, métodos, magnitud, características y evolución, Bogotá, D.C., Programa de las Naciones Unidas para el Desarrollo (PNUD).

(1992b): Pobreza: identificación y agregación, en L. Beccaria y otros, América Latina: el reto de la pobreza. Conceptos, métodos, magnitud, características y evolución, Bogotá, D.C., Programa de las Naciones Unidas para el Desarrollo (PNUD). (2001): Many faces of gender inequality, India's National Magazine, vol. 18, $\mathrm{N}^{\circ}$ 22, octubre-noviembre.

(2002): La desigualdad de género. La misoginia como problema de salud pública, Letras libres, abril.

UNIFEM (Fondo de Desarrollo de las Naciones Unidas para la Mujer) (2000): El progreso de las mujeres en el mundo, 2000, Nueva York. 